\title{
Analysis of the cellular expression of checkpoint molecules on immune cells in patients with breast cancer
}

\author{
Rawayeh Zarad \\ Zoology Department, Faculty of Science, Benha University, Benha, Egypt
}

\section{III}

Background: Breast cancer is a heterogeneous malignant tumor that includes several types of cells. Among them, only breast cancer stem cells (BCSCs) have tumorigenic ability. In this study we tested the hypothesis that BCSCs are behind disparities in behavior and biology of breast cancer between different ethnic groups and it is very important to investigate signaling pathways regulating BCSCs in each population. Therefore, we investigated the role of WNT, HH and apoptosis signaling pathways together with ALDH1A1 and CD44 in BCSCs resistance to chemotherapy in tumor biopsies collected from Egyptian patients compared to British patients. Aim: We investigated the expression levels of specific members of Wnt, Hedgehog, and apoptosis signaling pathways in BCSCs isolated from treated Egyptian and British patients. The change in their expression levels between the two populations was detected. Materials and Methods: Breast Cancer tumors were collected from Egyptian and British patients, dissociated to a single cell suspension in collagenase IV containing buffer. CSCs were isolated using anoikis resistance assay from treated and untreated samples. RNA was extracted and CDNA was synthesized. Custom qPCR arrays were used to detect the gene expression of Wnt5A, CXCR4, LEF1, TCF4, SHH, GLI1, BCL2, XIAP, ALDH1A1and CD44. Data were analyzed by $\Delta \Delta C$ t method. Results: Our data showed a number of genes with different expression levels in the treated Egyptian patients compared to the treated British patients. Conclusion: Molecular mechanisms that regulate BCSCs differ from a population to another. Moreover, the different treatment regimens in different countries may affect the way BCSCs resist therapy.

Keywords: Breast Cancer Stem Cells; Hedge Hog; Wnt 